\title{
Efecto analgésico preoperatorio del bloqueo PENG en pacientes con fractura de cadera
}

\section{Preoperative Analgesic efects of PENG block in hip fracture}

\author{
Elena Simón Polo1,* \\ 1 Anestesia y Reanimación, Hospital General de Albacete. España.
}

Conflicto de intereses: Ninguno.

Fecha de recepción: 03 de marzo de 2021 / Fecha de aceptación: 12 de mayo de 2021

\begin{abstract}
Hip fractures are a very common and a highly prevalent pathology in our setting, being one of the main reasons for visit in the emergency room. Regional analgesia in patients with hip fracture is a procedure frequently performed in the perioperative period since it allows the patient to offer adequate analgesia as well as a decrease in the consumption of intravenous opioids and their derived secondary effects. In recent years, epidural analgesia, femoral nerve block and fascia iliac blocks have been the post used techniques in hip fracture analgesia, achieving motor and sensory block in most patients, sometimes making initial ambulation later due to a weak of the psoas muscle. Recently, several anatomical studies have highlighted the importance of the sensory innervation of the articular hip inervation, developing the PENG approach and describing it as a more complete technique with better analgesic results than the approaches used before. In this block, an absence of motor weakness has been reported. We present four clinical cases of patients with hip fracture who underwent PENG block; the approach and the technique used are exposed, as well as the results obtained in terms of analgesia, valued using the visual analógic scale (EVA). This approach offers promising results, so it can be very useful in providing quality analgesia in patients with hip fractures in the preoperative period. Further studies may be interesting to validate the efficacy and superiority of the PENG blocks over the conventional techniques described.
\end{abstract}

Key words: Hip fracture, pericapsular nerve group block, regional anesthesia, ultrasound.

\section{RESUMEN}

Las fracturas de cadera constituyen una patología muy frecuente y de gran prevalencia en nuestro medio, siendo uno de los principales motivos de consulta en urgencias. La analgesia regional en pacientes con fractura de cadera es un procedimiento frecuentemente realizado en el periodo perioperatorio ya que permite ofrecer una adecuada analgesia al paciente así como una disminución del consumo de opioides intravenosos y sus efectos secundarios derivados. En los últimos años, la analgesia epidural, el bloqueo del nervio femoral así como los bloqueos de la fascia ilíaca han sido las técnicas más empleadas en la analgesia de fractura de cadera, consiguiendo un bloqueo motor y sensitivo en la gran mayoría de los pacientes, y en ocasiones dificultando la deambulación inicial debido a una debilidad del músculo psoas. Recientemente, varios estudios anatómicos han destacado la importancia de la inervación sensitiva de las ramas articulares de la cadera, desarrollándose así el abordaje PENG y describiendo como un abordaje más completo y con mejores resultados analgésicos que los abordajes empleados hasta entonces como el bloqueo del nervio femoral o el bloqueo de la fascia ilíaca. En este tipo de bloqueos ha sido descrita una ausencia de debilidad motora. A continuación, se exponen cuatro casos clínicos de pacientes que presentaron fractura de cadera a los que se les realizó el bloqueo PENG de forma preparatoria; en el caso se expone el abordaje y la técnica empleados así como los resultados obtenidos en cuanto a analgesia se refiere, valorada mediante la escala visual analógica (EVA). Este abordaje ofrece resultados prometedores, por lo que puede ser de gran utilidad para proporcionar una analgesia de calidad en pacientes con fracturas de cadera en el período preoperatorio. La realización de estudios con mayor número de pacientes incluidos puede resultar de interés para poder validar la eficacia y superioridad de los bloques PENG sobre las técnicas convencionales descritas.

Palabras clave: Fractura de cadera, bloqueo del grupo de nervios pericapsulares, anestesia regional, ultrasonidos. 


\section{Introducción}

a cirugía de reemplazo articular es una de las cirugías más frecuentes en nuestro medio. En EEUU se estima que aproximadamente se realizan 1.000 .000 de artroplastias de cadera y rodilla de forma anual[1]. La fractura de cadera constituye una urgencia ortopédica en el anciano, asociándose con importante morbilidad y mortalidad[2].

El dolor tras una fractura de cadera resulta intenso e incapacitante para el paciente, por lo que es imprescindible proporcionar una analgesia adecuada que facilite una terapia física agresiva y de rehabilitación, evitando retrasos en el alta del hospital y disminuyendo el riesgo de tromboembolismo[3].

En los últimos años, la realización de bloqueos nerviosos periféricos en combinación con analgesia intravenosa ha cobrado relevancia clínica en el manejo analgésico perioperatorio y postoperatorio[4].

El bloqueo del nervio femoral[5] y el bloqueo del compartimento de la fascia iliaca (FICB)[5] han sido estrategias analgésicas clásicamente empleadas en la analgesia en las fracturas de cadera, debido fundamentalmente a la reducción del consumo de opioides intravenosos, así como los efectos secundarios derivados[7].

Sin embargo, estos bloqueos pueden resultar incompletos o bien ofrecer una analgesia leve moderada debido a que la articulación se encuentra inervada por otras estructuras nerviosas adicionales, como el nervio obturador[8].

El bloqueo del grupo de nervios pericapsular (PENG) constituye una técnica analgésica que consiste en la infiltración del anestésico local en el plano musculofascial entre el músculo psoas y la rama púbica superior[9]. Este bloqueo se ha descrito recientemente como una opción eficaz para la analgesia de la cadera, ya que se dirige a las ramas articulares que inervan la cadera, por lo que podría ser de gran utilidad para la analgesia durante el perioperatorio.

\section{Caso clínico}

Presentamos el caso de cuatro pacientes ingresados de forma urgente en la planta de traumatología con diagnóstico de fractura subcapital de cadera que presentaron dolor periopera- torio con indicación de corrección quirúrgica mediante osteosíntesis.

Las edades de estos pacientes eran comprendidas entre 72 y 84 años $(72,75,80$ y 82). Ninguno de los pacientes presentaban alergias medicamentosas y ningún paciente presentaba alteraciones analíticas que pudiesen contraindicar una técnica locorregional (coagulopatía, plaquetopenia). Ninguno de los pacientes presentaba neurópata en la extremidad a estudio.

Dentro de la primera hora de ingreso en planta de hospitalización (aproximadamente una hora desde la llegada a urgencias) se explicó a cada paciente la posibilidad de realizar una técnica analgésica regional previo al procedimiento quirúrgico, minimizando así los efectos secundarios derivados de fármacos opioides intravenosos.

Previamente, se realizó una valoración preoperatoria y se obtuvo el consentimiento informado por escrito de todos los pacientes. Se midió el grado de dolor inicial medido por la escala visual analógica (EVA) durante la movilización en camilla y posicionamiento para la anestesia.

Tras colocar al paciente en posición supina, se empleó una sonda ecografica curvilínea de baja frecuencia (2-5 MHz). La sonda se colocó paralela al pliegue inguinal, a nivel de espina ilíaca anterosuperior, alineándose posteriormente con la rama púbica girando la sonda en sentido antihorario (aproximadamente 45 grados). A continuación, se identificaron la eminencia iliopúbica, el músculo iliopsoas con tendón prominente encima de la rama púbica, la arteria femoral y el músculo pectíneo (Figura 1).

Posteriormente, se introdujo una aguja de $80 \mathrm{~mm}$ estándar $22 \mathrm{G}$ hipereicoica y se administró una dosis de 100 miligramos de ropivacaína 0,5\% con $4 \mathrm{mg}$ de dexametasona (volumen total $20 \mathrm{ml}$ ) utilizando una técnica en plano guiada ecográficamente, depositando el anestésico local por debajo del tendón del psoas.

Se evaluaron las puntuaciones de dolor en reposo y en sedestación mediante la escala visual analógica (EVA) a los 20 minutos después de la ejecución del procedimiento (Figura 2).

Todos los pacientes a estudio presentaban dolor significativo antes del bloqueo PENG. La puntuación máxima en la EVA fue de 9, mientras que la mínima fue de 6.

Unos 20 minutos tras el bloqueo, revaluamos a los pacientes solicitándoles que flexionaran la cadera. Todos los pacientes

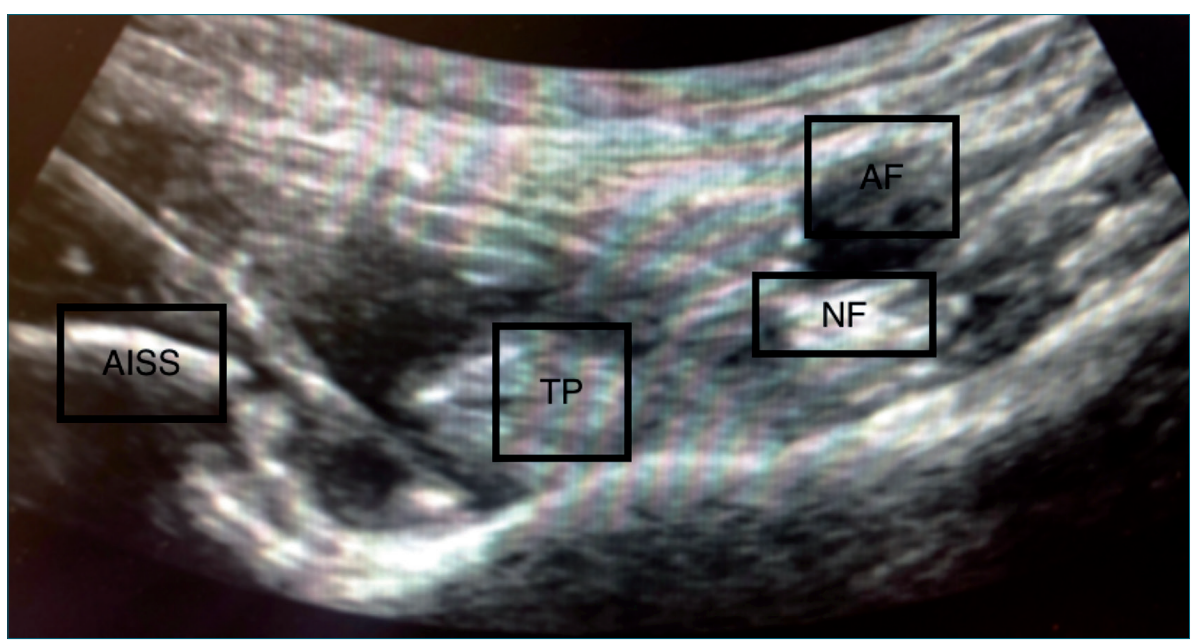

Figura 1. Imagen ecográfica de las estructuras con sonda convex. Abreviaturas: AF: Arteria Femoral; NF: Nervio femoral; TP: Tendón del Psoas; AISS: Espina ilíaca antero superior. 


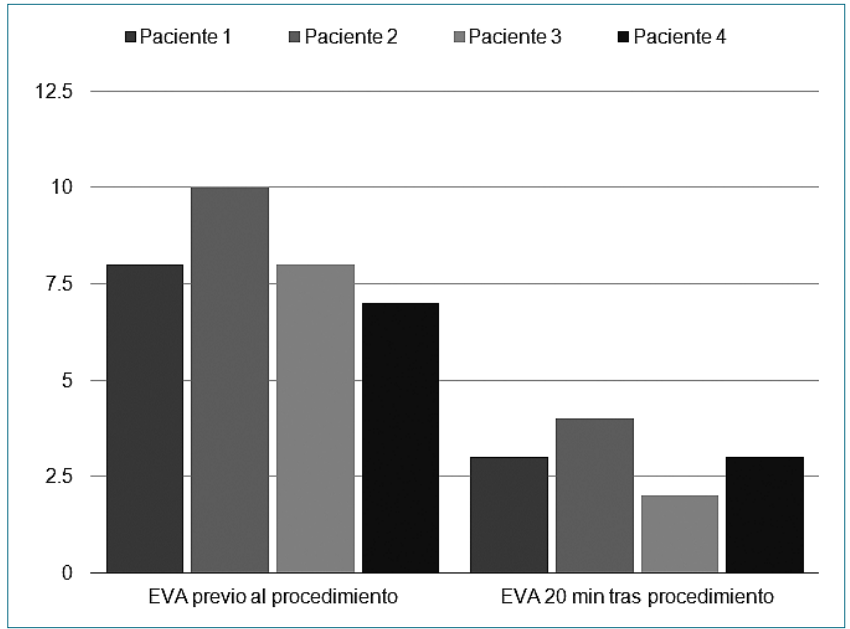

Figura 2. Se observa la disminución del dolor evaluado por el EVA luego de realizado el bloqueo.

fueron capaces de flexionar la cadera y las puntuaciones de EVA se redujeron tanto en decúbito supino como durante el movimiento activo de la extremidad. Tres pacientes presentaron una puntuación de 3 y un paciente de 2 .

Ninguno de los pacientes presentó debilidad en el músculo cuádriceps.

Todas las intervenciones se realizaron durante las primeras $12-24$ h y la media de la duración del bloqueo realizado fue de $12 \mathrm{~h}$.

\section{Discusión}

Las técnicas de analgesia regional guiadas ecográficamente son cada vez más utilizadas en el tratamiento del dolor de pacientes con fracturas de cadera, ya que proporcionan una adecuada analgesia y una disminución del uso de opioides intravenosos con una reducción de los efectos secundarios derivados de su uso[7].

En los últimos años, han sido múltiples las publicaciones destinadas a conocer mejor la inervación sensitiva de la cadera. Esta idea ha llevado al abordaje del bloqueo de las ramas de los nervios pericapsulares (PENG), con la intención de bloquear únicamente las ramas articulares sensitivas.

La cápsula anterior de la cadera se encuentra inervada por tres nervios: nervio femoral, nervio obturador y nervio obturador accesorio. Las ramas articulares del nervio femoral y del nervio obturador accesorio comparten referencias anatómicas comunes en lo profundo en el tendón del psoas, entre la espina iliaca inferior y la eminencia iliopectinea, base anatómica para el bloqueo PENG[10].

Otros estudios han mostrado que tanto en los bloqueos de la fascia ilíaca como en el bloqueo 3 en 1, el anestésico local no viaja tan median como para llegar al nervio obturador a nivel de L5, siendo poco probable que se alcancen las ramas articulares del nervio femoral[11].

\section{Conclusiones}

El bloqueo PENG proporciona una analgesia de cadera completa dado que cubre las principales ramas articulares que inervan la articulación de la cadera, siendo eficaz para reducir tanto dolor en reposo, en movimiento y permitiendo la sedestación. A pesar de que también existen otras técnicas regionales que proporcionan una analgesia similar, como es el caso de los bloqueos femorales y obturadores; sin embargo dichas técnicas se asocian con mayor bloqueo motor asociado, pudiendo dificultar la recuperación e inicio de la deambulación tras una intervención quirúrgica.

Los resultados de esta serie de casos son prometedores y puede ser de gran utilidad para proporcionar una analgesia de calidad en pacientes con fracturas de cadera durante el preoperatorio. Sin embargo, serían preciso estudios con mayor muestra poblacional para poder validar la eficacia y superioridad de los bloques PENG sobre las técnicas convencionales descritas.

La presente investigación no ha recibido ayudas específicas provenientes de agencias del sector público, sector comercial o entidades sin ánimo de lucro.

Responsabilidades éticas: Los autores declaran que para esta investigación no se han realizado experimentos en seres humanos ni animales.

Confidencialidad de los datos: Los autores declaran que han seguido los protocolos de su centro de trabajo sobre la publicación de datos de pacientes.

\section{Referencias}

1. Singer BR, McLauchlan GJ, Robinson CM, Christie J. Epidemiology of fractures in 15,000 adults: the influence of age and gender. J Bone Joint Surg Br. 1998 Mar;80(2):243-8. https://doi. org/10.1302/0301-620X.80B2.0800243 PMID:9546453

2. Brauer $C A$, Coca-Perraillon M, Cutler DM, Rosen AB. Incidence and mortality of hip fractures in the United States. JAMA. 2009 Oct;302(14):1573-9. https://doi.org/10.1001/jama.2009.1462 PMID:19826027

3. Mejía-Terrazas GE, Peña-Riveron A, Unzueta-Navarro D. Analgesia postoperatoria en cirugía de reemplazo articular. Acta Ortop Mex. 2013 Jul-Aug;27(4):273-8. PMID:24707619

4. Guay J, Kopp S. Peripheral nerve blocks for hip fractures in adults. Cochrane Database Syst Rev. 2020 Nov;11(4):CD001159. https://doi.org/10.1002/14651858.CD001159.pub3 PMID:33238043

5. Unneby A, Svensson O, Gustafson Y, Olofsson B. Femoral nerve block in a representative sample of elderly people with hip fracture: A randomised controlled trial. Injury. 2017 Jul;48(7):1542-9. https://doi.org/10.1016/j.injury.2017.04.043 PMID:28501287

6. Foss NB, Kristensen BB, Bundgaard M, Bak M, Heiring C, Virkelyst $C$, et al. Fascia iliaca compartment blockade for acute pain control in hip fracture patients: a randomized, placebocontrolled trial. Anesthesiology. 2007 Apr;106(4):773-8. https://doi.org/10.1097/01.anes.0000264764.56544.d2 PMID:17413915

7. Morrison RS, Magaziner J, Gilbert M, Koval KJ, McLaughlin MA, Orosz G, et al. Relationship between pain and opioid analge- 
sics on the development of delirium following hip fracture. J Gerontol A Biol Sci Med Sci. 2003 Jan;58(1):76-81. https://doi. org/10.1093/gerona/58.1.M76 PMID:12560416

8. Wertheimer LG. The sensory nerves of the hip joint. J Bone Joint Surg Am. 1952 Apr;34-A(2):477-87. https://doi. org/10.2106/00004623-195234020-00016 PMID:14917714

9. Girón-Arango L, Peng PW, Chin KJ, Brull R, Perlas A. Pericapsular Nerve Group (PENG) Block for Hip Fracture. Reg Anesth Pain Med. 2018 Nov;43(8):859-63. https://doi.org/10.1097/

\section{AAP.0000000000000847 PMID:30063657}

10. Zaragoza-Lemus G, Portela-Ortiz JM, Díaz-Guevara G. Blockade of the pericapsular nerve group (PENG) for hip surgery. Revista Mexicana de Anestesiologia. 2020;43(1):69-72. https://doi. org/10.35366/CMA201M.

11. Acharya U, Lamsal R. Pericapsular Nerve Group Block: An Excellent Option for Analgesia for Positional Pain in Hip Fractures. Case Rep Anesthesiol. 2020 Mar;2020:1830136. https://doi. org/10.1155/2020/1830136 PMID:32231802 